\title{
BMJ Global Health Understanding for whom, why and in what circumstances payment for performance works in low and middle income countries: protocol for a realist review
}

\author{
Josephine Borghi, ${ }^{1}$ Neha S Singh, ${ }^{1}$ Garrett Brown, ${ }^{2}$ Laura Anselmi, ${ }^{3}$ \\ Soren Kristensen ${ }^{4}$
}

To cite: Borghi J, Singh NS, Brown G, et al. Understanding for whom, why and in what circumstances payment for performance works in low and middle income countries: protocol for a realist review. BMJ Glob Health 2018;3:e000695. doi:10.1136/ bmjgh-2017-000695

Handling editor Seye Abimbola

- Additional material is published online only. To view please visit the journal online (http://dx.doi.org/10.1136/ bmjgh-2017-000695)

Received 5 January 2018 Revised 3 May 2018 Accepted 17 May 2018

\section{Check for updates}

${ }^{1}$ Department of Global Health and Development, London School of Hygiene and Tropical Medicine, London, UK ${ }^{2}$ School of Politics and International Studies, University of Leeds, Leeds, UK

${ }^{3}$ Manchester Centre for Health Economics, University of Manchester, Manchester, UK ${ }^{4}$ Faculty of Medicine, Institute of Global Health Innovation, Centre for Health Policy, Imperial College, London, UK

Correspondence to Dr Josephine Borghi; jo.borghi@Ishtm.ac.uk

\section{ABSTRACT \\ Background Many low and middle income countries} (LMIC) are implementing payment for performance (P4P) schemes to strengthen health systems and make progress towards universal health coverage. A number of systematic reviews have considered P4P effectiveness but did not explore how P4P works in different settings to improve outcomes or shed light on pathways or mechanisms of programme effect. This research will undertake a realist review to investigate how, why and in what circumstances P4P leads to intended and unintended outcomes in LMIC. Methods Our search was guided by an initial programme theory of mechanisms and involved a systematic search of Medline, Embase, Popline, Business Source Premier, Emerald Insight and EconLit databases for studies on P4P and health in LMIC. Inclusion and exclusion criteria identify literature that is relevant to the initial programme theory and the research questions underpinning the review. Retained evidence will be used to test, revise or refine the programme theory and identify knowledge gaps. The evidence will be interrogated by examining the relationship between context, mechanisms and intended and unintended outcomes to establish what works for who, in which contexts and why.

Discussion By synthesising current knowledge on how P4P affects health systems to produce outcomes in different contexts and to what extent the programme design affects this, we will inform more effective P4P programmes to strengthen health systems and achieve sustainable service delivery and health impacts.

\section{BACKGROUND}

There has been growing international commitment in recent years to the goal of achieving universal health coverage (UHC) or access to affordable and effective care. UHC relies on strong health systems ${ }^{1}$ and on the effective and equitable allocation and use of available resources. However, health systems in many settings struggle to deliver quality care due, for example, to inadequate
Summary box

Previous reviews of payment for performance (P4P) have focused on programme effectiveness, finding mixed effects on incentivised outcomes. These reviews did not systematically examine how P4P works in different contexts and its potential unintended effects.

- This article presents the protocol for a realist review to shed light on for who, how and in what circumstances P4P works in low and middle income countries (LMIC). This review will increase our understanding of how P4P affects health systems and how health systems can be strengthened to deliver better care and key attributes of successful P4P programmes in different contexts.

- The outcome of the review will be a refined middle range programme theory reflecting actor response to P4P (mechanisms) and contextual factors shaping this, that can be generalised across LMIC settings.

- The review will inform the design of more effective P4P programmes to strengthen health systems for specific contexts and minimise unintended negative effects.

infrastructure; lack of motivated staff; a lack of drugs and medical supplies and limited adherence to clinical care guidelines. ${ }^{2}$

Payment for performance (P4P) has been proposed as a strategy to strengthen health systems, to improve service delivery and population health. P4P consists of financial incentives to healthcare providers and/or their managers based on service delivery performance $^{34}$ and assumes individuals exert more effort in response to incentives, which will result in improved quality of care. $\mathrm{P} 4 \mathrm{P}$ has been widely applied in the USA and the $\mathrm{UK}^{5-7} \mathrm{In}$ these countries, payers most often introduce $\mathrm{P} 4 \mathrm{P}$ by targeting the organisational level rather 
than the individual provider level, with a view to improving quality of care. Literature from these settings suggests that while P4P may improve processes of care, very few studies have demonstrated improvements in health outcomes. ${ }^{8}$ Calls for a better understanding of $\mathrm{P} 4 \mathrm{P}$ mechanisms have also been voiced ${ }^{910}$ and some authors are now suggesting a reconsideration of the role of $\mathrm{P} 4 \mathrm{P}$ as a means of improving outcomes in healthcare. ${ }^{11}$ Over 30 low and middle income countries (LMIC) are currently implementing P4P schemes in the health sector with financial support from international donors. ${ }^{12}$ In LMIC, P4P can involve additional resources to improve service delivery, investments in information systems, more frequent supervision of healthcare providers and greater financial decentralisation. ${ }^{13}$ Hence, in these settings, $\mathrm{P} 4 \mathrm{P}$ represents a complex package of interventions, aimed at strengthening health systems to deliver better care. ${ }^{14}$ The focus of incentives in these settings is on the quantity of services delivered and quality of care.

Most early evaluations of P4P schemes in LMIC considered programme effectiveness in terms of improving patient and population health outcomes. A systematic review of the effect of $\mathrm{P} 4 \mathrm{P}$ programmes in LMIC published in 2012 reported that the evidence base was too limited to draw overall conclusions and that more attention was needed to understand how incentive design impacts on programme effectiveness. ${ }^{3}$ This review is currently being updated. A more recent review of the effect of $\mathrm{P} 4 \mathrm{P}$ on quality found evidence of improved process quality for antenatal care, but limited or mixed evidence for other quality indicators. ${ }^{15} \mathrm{~A}$ third review examined the effects of P4P on HIV indicators, identifying only four studies with positive effects on coverage of HIV testing for couples and pregnant women and coverage of antiretrovirals in pregnant women, infants and adults. ${ }^{16}$

In more recent years, researchers have recognised the importance of also understanding change mechanisms ${ }^{17}$ and documenting the effects of $\mathrm{P} 4 \mathrm{P}$ on the health system. As a result, there is growing evidence of the effects of P4P on health workers, ${ }^{18-24}$ accountability mechanisms including supervision systems and community engagement in service delivery, ${ }^{25} 26$ information systems and use of data, ${ }^{27}$ drug availability, ${ }^{28}$ patient satisfaction and provider-patient interactions. ${ }^{5}$ None of this evidence is captured within the systematic reviews referenced above. A more recent review did summarise much of this evidence $;{ }^{13}$ however, the review was focused on extracting policy recommendations, but did not explore how $\mathrm{P} 4 \mathrm{P}$ works in different settings to bring about improvements in outcomes or shed light on the pathways or mechanisms of programme effect.

In light of emerging evidence of the health system effects of $\mathrm{P} 4 \mathrm{P}$, a further review of the literature is needed to investigate how, why and in what circumstances $\mathrm{P} 4 \mathrm{P}$ leads to intended and unintended outcomes in LMIC and how the design of $\mathrm{P} 4 \mathrm{P}$ incentives affects the way $\mathrm{P} 4 \mathrm{P}$ programmes work and their outcomes. Such a review will increase our understanding of how P4P affects health systems and how health systems can be strengthened to deliver better care and key attributes of successful P4P programmes in different contexts.

The choice of synthesis methodology should be driven by the research question ${ }^{29-32}$ and we contend that a realist approach is best suited for such a review. A systematic review is a robust methodology for assessing whether interventions are effective, but is not suited to unpacking or explaining effects or to answering 'how and why' questions. ${ }^{33}{ }^{34}$ Indeed, the existing systematic reviews on P4P only included experimental or quasi-experimental studies addressing the question of how effective the programme was and did not document process changes or contextual factors which allowed the interventions to produce outcomes. In contrast to systematic reviews, realist reviews are able to include and synthesise a much broader set of evidence, including qualitative methods which address 'how and why' questions. A realist approach assumes that complex interventions do not operate in a silo; rather they operate within social systems and it is 'the mechanism' (the response and behaviour of agents interacting within the social system) that determines outcomes within a given context. ${ }^{34} 35$ The context can be social, cultural, historical or institutional and is what facilitates or limits the action of agents. ${ }^{36}$ The realist approach is guided by an initial programme theory of how the programme is expected to lead to given outcomes, why and in what context (referred to as a context-mechanism-outcome configuration-CMO). The realist approach tries to then empirically test the hypothesised 'mechanisms' or the way actors are expected to respond to $\mathrm{P} 4 \mathrm{P}$ programmes and the changes their response brings about that leads to intended and unintended outcomes and to assess how this mechanism varies according to the context of programme implementation. The review findings are then used to determine which CMO configuration(s) offer the most robust and plausible explanation of observed outcomes. This resulting CMO configuration is then compared with the initial programme theory, which is modified in light of these findings, resulting in a 'middle range' programme theory (or a robust understanding of the sets of mechanisms and how they unfold in different contexts), which can be generalised across LMIC. ${ }^{37}$ In this way, the realist approach aims to discern what works for whom, in what context and how and why it works to produce both intended and unintended outcomes. ${ }^{37}$

\section{METHODS}

\section{Aim and objectives}

The overall aim of this review is to help researchers and policy makers understand how and why $\mathrm{P} 4 \mathrm{P}$ programmes implemented in low and middle income countries result in intended or unintended outcomes, how the context within which they are implemented affects this and which incentive designs are most effective. In so doing, the review will produce a refined middle range programme theory for $\mathrm{P} 4 \mathrm{P}$ reflecting the way the programme works to deliver specific outcomes 


\begin{tabular}{|c|c|c|}
\hline & Steps & Task(s) \\
\hline 1 & $\begin{array}{l}\text { Clarifying the initial } \\
\text { programme theory }\end{array}$ & $\begin{array}{l}\text { Search for initial theories and } \\
\text { then consult with experts }\end{array}$ \\
\hline 2 & Search strategy & $\begin{array}{l}\text { Search electronic databases } \\
\text { using keywords and Medical } \\
\text { Subject Heading (MeSH) terms }\end{array}$ \\
\hline 3 & $\begin{array}{l}\text { Select and } \\
\text { appraise } \\
\text { documents }\end{array}$ & $\begin{array}{l}\text { Use inclusion and exclusion } \\
\text { criteria to screen for relevant } \\
\text { abstracts, articles and } \\
\text { reports } \\
\text { Retrieve full-text of articles } \\
\text { and reports }\end{array}$ \\
\hline 4 & Extract data & $\begin{array}{l}\text { Use standardised tool to } \\
\text { extract relevant data } \\
\text { Search reference lists } \\
\text { by hand for additional } \\
\text { potentially relevant articles } \\
\text { and reports }\end{array}$ \\
\hline 5 & $\begin{array}{l}\text { Analysis and } \\
\text { synthesis process }\end{array}$ & $\begin{array}{l}\text { Analyse data for content } \\
\text { and outcome patterns and } \\
\text { synthesise mechanisms } \\
\text { NB: Realist reviews follow } \\
\text { an iterative search process, } \\
\text { so revise Step } 2 \text { (ie, search } \\
\text { strategy) if relevant }\end{array}$ \\
\hline 6 & $\begin{array}{l}\text { Present and } \\
\text { disseminate } \\
\text { revised programme } \\
\text { theory }\end{array}$ & $\begin{array}{l}\text { Present and refine revised } \\
\text { theoretical findings with } \\
\text { relevant stakeholders and } \\
\text { experts }\end{array}$ \\
\hline
\end{tabular}

in different contexts that can be generalised across LMIC. The review aims to address the following specific questions:

1. How do actors respond to $\mathrm{P} 4 \mathrm{P}$ programmes and what changes does their response bring about that leads to intended and unintended outcomes (what is the 'mechanism' through which the programme affects outcomes)?

2. What contextual and programme design factors determine whether the identified 'mechanisms' produce these outcomes?

\section{Study design}

We will carry out a realist review as proposed by Pawson and colleagues. ${ }^{37}$ The study will be conducted in six steps, namely: (1) developing initial programme theory; (2) searching for evidence; (3) selecting and appraising documents; (4) extracting data; (5) synthesising evidence; (6) presenting and disseminating middle range programme theory, as outlined in table 1 and detailed below. At the time of writing, some of the first steps had already been initiated or completed. As a result, we use the past tense to describe steps that have been completed and the future tense to describe steps that have yet to start.

\section{Step 1-Developing an initial programme theory}

This step aims to develop an initial programme theory for P4P: (1) highlighting the anticipated response of actors to the $\mathrm{P} 4 \mathrm{P}$ programme and how this response translates into (intended and unintended) changes in outcomes and (2) identifying the contextual factors ( institutional, organisational, socioeconomic, cultural) influencing the actor response to the programme (the 'mechanism'). This step also aims to present this theory visually within a diagram. To develop an initial programme theory for P4P, we drew on five sources of information: (1) motivation theories and theories of demand; (2) existing published theories of change related to $\mathrm{P} 4 \mathrm{P}$ that were known to the authors; (3) theories of change developed during stakeholder workshops convened by the research team; (4) the research team's (JB, GB, LA) own research knowledge and experience related to $\mathrm{P} 4 \mathrm{P}$; (5) existing reviews of $\mathrm{P} 4 \mathrm{P}$.

Existing bodies of theory were used to support the general development of the initial programme theory in relation to provider response to incentives (motivation theories of how workers respond to incentives ${ }^{38-40}$ ) and patient response to changes in service provision (the Grossman theory of demand ${ }^{41}$ ). To tailor the initial programme theory more specifically to $\mathrm{P} 4 \mathrm{P}$, two published theories of change were then identified and appraised: that presented in the World Bank's P4P impact evaluation toolkit ${ }^{12}$ and a theory of change used within an evaluation study of $\mathrm{P} 4 \mathrm{P}$ in Tanzania, in which two of the researchers were involved (JB, LA). ${ }^{42}$ Two theories of change diagrams were also reviewed that resulted from stakeholder workshops: the first developed by researchers studying P4P in LMIC, who attended a Resilient and Responsive Health Systems research consortium workshop in Dar es Salaam, Tanzania in November 2015 (including JB and LA) and the second developed by Mexican and UK researchers (including JB and LA) studying financial incentives in health, during a Newton funded Researcher Links UK-Mexico workshop in April 2015. To derive our initial programme theory for this review and construct an associated diagram, two members of the review team (JB and NSS) appraised each diagram, giving priority to common pathways and considered these in relation to existing knowledge within the team and evidence from published reviews.

The resulting diagram, figure 1, provides a visual representation of our initial programme theory. In summary, the direct effect of individual financial incentives is to make health workers more motivated to adhere to the incentivised dimensions of the service (obtain training to provide this care, increasing knowledge) and to adopt strategies to attract patients to facilities for incentivised services (for quantity targets) to maximise incentive payments. ${ }^{43-45}$ Actions taken to comply with incentivised indicators may include increased adherence to the clinical care guidelines, making services more affordable (reducing informal charges, boosting insurance enrolment ${ }^{46}$ ), making services more available (longer opening 
IMPACT ON THE HEALTH SYSTEM

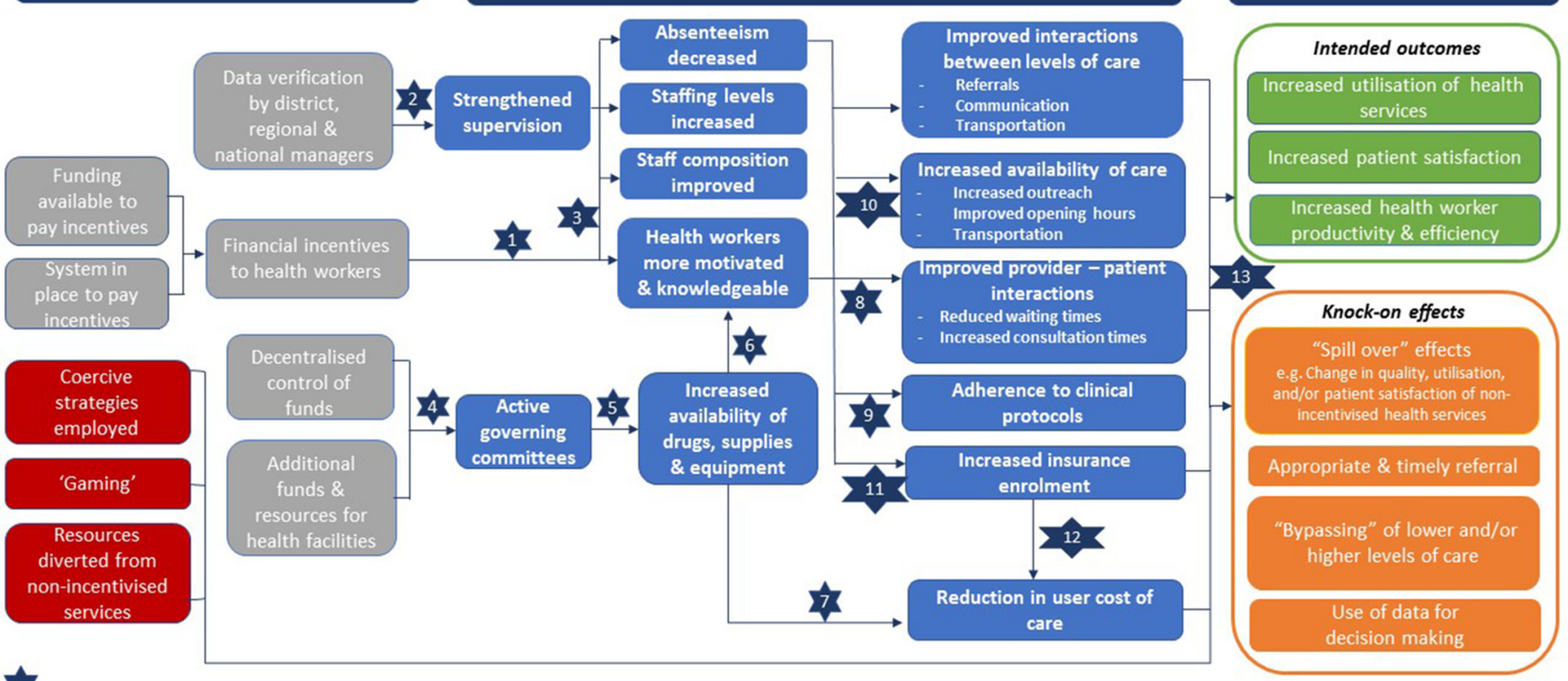

Assumptions: (1) Health workers become more motivated as a result of incentives; knowledge levels increase through investment in training to meet targets; or through re-allocation of staff to poor performing facilities; (2) Verification activities result in strengthened supervision; (3) Strengthened supervision motivates health workers; (4) Local bank accounts \& facility revenue give a role to governing committees; (5) Additional funds are used to procure drugs \& supplies through the committee; (6) Increased availability of drugs \& supplies increases motivation levels; (7) Increased availability of drugs \& supplies reduces user costs of care; (8) More
motivated health workers improve interactions with patients; (9) Increased motivation, knowledge \& availability of drugs increases adherence to clinical care guidelines; (10) To meet utilisation targets, health workers undertake more community outreach activities; facilities improve opening hours \& ensure transportation for referrals (11) Health workers encourage insurance enrolment to increase facility revenue; patients are more undertake more community outreach activities; facilities improve opening hours \& ensure transportation for referrals (11) Health workers encourage insurance enrolment to increase facility revenue; patients are more
willing to ioin due to better perceived auality of care: (12) Insurance coverage reduces user costs of care: (13) Qualitv imbrovements \& increased affordabilitv of care imbact incentivised \& non-incentivised outcomes

Figure 1 Initial programme theory mapping the mechanisms through which P4P affects the health system and results in outcomes.

hours; increased outreach care ${ }^{47}$ ) and becoming more responsive to patient needs (improved client-provider interactions $\left.{ }^{15}{ }^{46}\right)$, resulting in greater patient satisfaction, greater community engagement in service delivery through facility governing committees ${ }^{48}$ ). Other components of $\mathrm{P} 4 \mathrm{P}$ programmes are also expected to result in change. ${ }^{49}$ The need for managers to verify performance data may increase interactions between providers and managers, and when the latter are incentivised, strengthen relations between levels of the system, including referrals, and result in more frequent and focused supportive supervision. ${ }^{26}$ Greater commitment of managers to service delivery may increase the use of data for decision making and the prioritisation of resources to maximise performance on incentivised indicators (eg, through increased staffing levels and improved staff composition ${ }^{50}$ and facilitating provider access to drugs and supplies). Part of the incentive is provided to the facility for investment in drugs, medical supplies or equipment, the availability of which would conceivably increase in response to the programme ${ }^{28}$ and in turn would enhance worker motivation and ability to deliver better and more affordable care (by reducing user charges), increasing patient satisfaction and service utilisation. However, P4P can also result in unintended consequences such as misreporting performance (gaming), ${ }^{51} 52$ the use of coercive strategies to boost demand, ${ }^{47}$ a reluctance to refer or treat patients that could negatively affect their performance score, a displacement of effort away from un-incentivised services $^{53}$ and positive spillover effects. ${ }^{46}$
As contextual factors were not identified within the theory of change diagrams we reviewed and appraised, these were not included in our diagram. However, we identify a number of potentially relevant contextual factors within our initial programme theory, notably: other policies especially those operating on the demand side might impact on P4P effectiveness; the level of health system performance and capacity at the time of introduction of $\mathrm{P} 4 \mathrm{P}$ and access to other funding streams ${ }^{54}$; the organisational culture within which $\mathrm{P} 4 \mathrm{P}$ is introduced, management competencies; the wider context of health worker pay $^{54}$ and the population culture and attitudes towards formal medical care. ${ }^{55}$ We will also explore whether effects differ in fragile or postconflict settings, compared with other settings, ${ }^{3}$ and according to how $\mathrm{P} 4 \mathrm{P}$ was introduced (embedded in existing government purchasing systems or as a stand alone programme). We will look for details of context within the reviewed papers (in relation to characteristics of populations covered by $\mathrm{P} 4 \mathrm{P}$; providers implementing $\mathrm{P} 4 \mathrm{P}$ and the broader (institutional, economic, geographic, cultural) context within which it is implemented.

We will look for evidence of how contextual factors affect implementation and programme mechanisms and through comparison across studies, we will examine whether mechanisms and outcomes are associated with any dimensions of the context within which they are implemented. Some aspect of programme design have also been identified as potentially relevant to understanding mechanisms and outcomes (specifically the 
level of incentive relative to other funding, and who and what is and is not incentivised). ${ }^{355}$

The initial programme theory was presented to a policy and academic audience at the Fourth Global Forum for Human Resources in Health in November 2017 for external validation. The programme theory and diagram will be revisited throughout the evidence review process and revised to reflect emerging findings.

\section{Step 2-Searching for evidence}

In a second step, we conducted a systematic search for primary studies that are relevant to the programme theory set out in Step 1, with the aim of identifying evidence to test and refine the programme theory. We searched Medline, Embase, Popline, Business Source Premier, Emerlad Insight and EconLit databases. A search strategy was developed and carried out in collaboration with an experienced university librarian. The search included appropriate indexing terms (ie, MeSH terms and keywords) on P4P; on mechanisms relating to programme theory and geographic focus (eg, low and middle income countries as defined by the World Bank ${ }^{56}$ ). The search strategy was first developed in Medline and then adapted to the other databases (online supplementary appendix 1). The results of the initial search were reviewed to examine whether any known references were excluded. The search strategy was subsequently modified to be more inclusive and ensure the retention of all known articles of relevance by searching for $\mathrm{P} 4 \mathrm{P}$ $\mathrm{MeSH}$ terms and keywords and restricting to low and middle-income countries as defined by the World Bank. ${ }^{56}$ The search period covered 1 January 1995, when empirical literature first started to emerge on P4P in LMIC, to 21 November 2017.

After screening papers identified in the systematic search, we will also search for international unpublished and grey literature (eg, websites of key stakeholders including: World Bank, the World Health Organization, Cordaid, Norad, the Department for International Development, USAID and PEPFAR) as well as Google Scholar and Web of Science to identify academic working papers and evaluation reports or policy documents published by LMIC governments, international organisations, non-governmental organisations and consultancy firms. The research team's contacts with networks of researchers, decision-makers and other stakeholders with knowledge of P4P in LMIC will also facilitate the identification and collection of such documents.

Reference lists of all identified studies and documents will be screened to identify potential additional literature that were not captured within the original review. The literature review search will end at the point of saturation, that is, when the research yields no further new sources of information. All search results from electronic databases and other sources will be imported into Endnote reference management software and duplicates removed.
Step 3-Selecting and appraising documents

Inclusion and exclusion criteria were developed to identify literature that is relevant to the initial programme theory (figure 1) and the research questions underpinning the review ${ }^{57}$ - see table 2. Retained evidence will be used to test, revise or refine elements within the initial programme theory. The review will also identify knowledge gaps where evidence is limited in relation to the initial programme theory. Where there is conflicting evidence on a given component, we will explore contextual and scheme design differences that may account for variation in findings. Studies with any evaluation design were allowed as per realist review guidelines. The relevance of the retrieved articles/publications was assessed according to the inclusion and exclusion criteria outlined below. Next, two reviewers (NSS and JB) independently screened all titles and abstracts for suitability for inclusion. Disagreements were noted and discussed to reach agreement based on consistent criteria.

\section{Step 4-Extracting data}

Data will be extracted into a Microsoft Excel spreadsheet. The spreadsheet captures information on general study characteristics such as the author name, year, study setting, P4P implementing organisation, and study type. In addition, data will be extracted according to a set of domains identified by NSS and JB based on the content of the initial programme theory,documenting mechanisms (the programme effects on different health system elements and the links between these effects), context (who is this working for or not working for; why is this working or not working; in what contexts is it working/not working), outcomes (the final outcomes of the programme) and programme design elements (level of incentive, what is incentivised, who gets the incentive). To test the suitability of these domains they will be pretested on two purposefully selected articles by NSS and JB. The domains will be revised iteratively as the review progresses and new mechanisms and effects emerge from the evidence that were not within the original programme theory and as more focused and specific research questions arise.

We will also appraise the quality of methodology and describe the methodology using The Mixed-Method Appraisal Tool, which is suited to the assessment of quantitative, qualitative and mixed-methods approaches. ${ }^{58}$

The review will compare and contrast evidence, looking for consistent CMO configurations emerging from the evidence to support, refine or modify the initial programme theory.

\section{Step 5-Analysis and synthesis of data}

Each study will be read by two authors (JB and NSS) and discussed to assess whether emerging findings support, refute or reinterpret the preliminary programme theory (figure 1). To this end, evidence will be interrogated by examining the relationship between context, mechanisms and outcomes, both intended and unintended, 
Table 2 Inclusion and exclusion criteria

\section{Inclusion criteria}

The exposure (intervention) is a P4P intervention. These schemes had to target healthcare providers and/or managers (supply-side), incentives had to be financial and cash disbursement had to be varied accordingly to performance, defined as the achievement of quantitative indicators for selected healthcare services, quality-related indicators or both quantity-related and quality-related indicators. When performance is linked to quantitative outputs, it has to be related to 'selected healthcare services' as such criterion allows one to discriminate between P4P and fee for service mechanisms.

The study should isolate (or attempt to isolate) the effects of $\mathrm{P} 4 \mathrm{P}$ programmes from that of broader policy reforms, which often encompass the P4P reform.

The evaluation of pilot projects will be included in the study.

The study outcome has to be either a quantitative or qualitative measure (or both) of the impact of the P4P initiative on one or more health system functions described in the initial programme theory (figure 1) or other relevant mechanisms or contextual factors affecting outcomes.

\begin{tabular}{|c|c|}
\hline $\begin{array}{l}\text { The study should report on primary data sources. Where the } \\
\text { study refers to different sources of evidence for primary data } \\
\text { (eg, in the case of systematic reviews), the primary source of } \\
\text { information will be retrieved and explored. }\end{array}$ & $\begin{array}{l}\text { Studies that do not report primary data sources (eg, } \\
\text { commentaries or reviews), though these will be screened for } \\
\text { relevant additional references. }\end{array}$ \\
\hline $\begin{array}{l}\text { The intervention had to be implemented in an LMIC, as } \\
\text { defined by the World Bank. }\end{array}$ & Studies in high-income settings. \\
\hline Studies in English, French, Portuguese or Spanish. & $\begin{array}{l}\text { Studies in languages other than English, French, Portuguese } \\
\text { or Spanish }\end{array}$ \\
\hline
\end{tabular}
FFS, fee-for-service; LMIC, low and middle income countries; P4P, payment for performance.

to establish what works for who, in which contexts and why. The analysis and synthesis will involve looking for recurrent relationships between context, mechanisms and outcomes in the documents. We will examine how similar mechanisms act in different contexts to generate outcomes. The findings emerging from the review will be questioned and contradictory examples sought in the data. An appraisal of the strengths/weaknesses of the research methods used by papers to be integrated into the synthesis will be undertaken. The findings will be used to refine, adjust or modify theory, resulting in a middle-range theory ${ }^{37}$ of the links between contextual factors, mechanisms and outcomes of $\mathrm{P} 4 \mathrm{P}$ interventions.

\section{Step 6-Generating a revised programme theory}

The revised programme theory will be described in text and using a diagram and the review will be reported according to the standards outlined by the realist and meta-narrative evidence synthesis (RAMESES) group. $^{59}$ Pawson $e$ t $a l^{37}$ argue that stakeholders should be involved in confirming emerging findings and in dissemination activities. Emerging findings and CMO configurations will be presented and debated during a conference session on P4P theories of change in 2018. The final

\section{Exclusion criteria}

Schemes targeting beneficiaries of health services (ie, demand-side incentives); Supply-side P4P schemes outside of the health sector; P4P schemes that did not specifically target selective health services and, therefore, were not distinguishable from fee for service schemes

Studies which do not attempt to isolate the effects of P4P from broader reforms.

Studies of the 'potential' implementation of P4P strategies not yet in place.

Studies that only examine impact on ultimate service utilisation and health outcomes and do not examine mechanisms and/or context.

Studies that do not report primary data sources (eg, commentaries or reviews), though these will be screened for relevant additional references.

Studies in high-income settings.

Studies in languages other than English, French, Portuguese

programme theory will be that emerging from these stakeholder discussions.

\section{DISCUSSION}

This study involves a realist approach to synthesising evidence to generate an improved understanding of how and why $\mathrm{P} 4 \mathrm{P}$ programmes result in intended or unintended outcomes, within which contexts and which incentive designs are most effective. In so doing, the review will ultimately produce a refined middle range programme theory for $\mathrm{P} 4 \mathrm{P}$ reflecting actor response to $\mathrm{P} 4 \mathrm{P}$ (mechanisms) and contextual factors shaping these, that can be generalised across LMIC. The use of a realist approach will allow the review to describe and explain how and why P4P initiatives work (or fail to work) in different contexts by exploring the underlying programme theories and the interactions between contextual factors, mechanisms of change and outcomes.

Synthesising current knowledge of how P4P affects health systems to produce outcomes in different contexts and to what extent the incentive design affects this will inform more effective $\mathrm{P} 4 \mathrm{P}$ programmes to strengthen health systems and achieve sustainable service delivery 
and health impact and minimise unintended effects. This review will also shed light on how context shapes the design of performance-based financing programmes and their subsequent implementation, which will be useful in determining where $\mathrm{P} 4 \mathrm{P}$ can be most effectively implemented.

Contributors All authors contributed to conceptualising the protocol and writing the paper. JB wrote the first draft of the paper.

Funding This research was supported by a Health Systems Research Initiative grant number MR/P014429/1 jointly funded by the Medical Research Council, Wellcome Trust, Economic and Social Research Council, Department for International Development. SK was also funded by the NIHR Imperial Patient Safety Translational Research Centre.

Disclaimer The views expressed are those of the author(s) and not necessarily those of the funders.

Competing interests None declared.

Patient consent Not required.

Provenance and peer review Not commissioned; externally peer reviewed.

Data sharing statement № additional data are available.

Open access This is an open access article distributed in accordance with the terms of the Creative Commons Attribution (CC BY 4.0) license, which permits others to distribute, remix, adapt and build upon this work, for commercial use, provided the original work is properly cited. See: http://creativecommons.org/ licenses/by/4.0/

(C) Article author(s) (or their employer(s) unless otherwise stated in the text of the article) 2018. All rights reserved. No commercial use is permitted unless otherwise expressly granted.

\section{REFERENCES}

1. Sambo LG, Kirigia JM. Investing in health systems for universal health coverage in Africa. BMC Int Health Hum Rights 2014;14:28.

2. World Health Organization. What is quality of care and why is it important? http://www.who.int/maternal_child_adolescent/topics/ quality-of-care/definition/en/.

3. Witter S, Fretheim A, Kessy FL, et al. Paying for performance to improve the delivery of health interventions in low- and middleincome countries. Cochrane Database Syst Rev 2012:CD007899.

4. Musgrove P. Financial and other rewards for good performance or results: a guided tour of concepts and terms and a short glossary, 2011.

5. Campbell SM, Reeves D, Kontopantelis E, et al. Effects of pay for performance on the quality of primary care in England. $N$ Engl J Med 2009;361:368-78.

6. Sutton M, Nikolova S, Boaden R, et al. Reduced mortality with hospital pay for performance in England. $N$ Engl J Med 2012;367:1821-8.

7. Mendelson A, Kondo K, Damberg C, et al. The Effects of Pay-forPerformance Programs on Health, Health Care Use, and Processes of Care. Ann Intern Med 2017;166:341-53.

8. Mendelson A, Kondo K, Damberg C, et al. The Effects of Pay-forPerformance Programs on Health, Health Care Use, and Processes of Care: A Systematic Review. Ann Intern Med 2017;166:341-53.

9. Roland M. Pay-for-performance: not a magic bullet. Ann Intern Med 2012;157:912-3.

10. Epstein AM. Will pay for performance improve quality of care? The answer is in the details. N Engl J Med 2012;367:1852-3.

11. Frakt $A B$, Jha AK. Face the Facts: We Need to Change the Way We Do Pay for Performance. Ann Intern Med 2018;168:291-2.

12. Vermeersch $\mathrm{C}$, Rothenbuhler E, Sturdy J. Impact evaluation toolkit: measuring the impact of results-based financing on maternal and child health. Washington, DC: World Bank, 2012:321.

13. Renmans D, Holvoet N, Orach CG, et al. Opening the 'black box' of performance-based financing in low- and lower middleincome countries: a review of the literature. Health Policy Plan 2016;31:1297-309.

14. Medical Research Council. Developing and evaluating complex interventions: new guidance. 2008 http://www.mrc.ac.uk/ documents/pdf/complex-interventions-guidance/;.
15. Das A, Gopalan SS, Chandramohan D. Effect of pay for performance to improve quality of maternal and child care in low- and middleincome countries: a systematic review. BMC Public Health 2016;16:321.

16. Suthar AB, Nagata JM, Nsanzimana S, et al. Performance-based financing for improving HIV/AIDS service delivery: a systematic review. BMC Health Serv Res 2017;17:6.

17. Ssengooba F, McPake B, Palmer N. Why performance-based contracting failed in Uganda--an "open-box" evaluation of a complex health system intervention. Soc Sci Med 2012;75:377-83.

18. Shen GC, Nguyen HT, Das A, et al. Incentives to change: effects of performance-based financing on health workers in Zambia. Hum Resour Health 2017;15:20.

19. Bhatnagar A, George AS. Motivating health workers up to a limit: partial effects of performance-based financing on working environments in Nigeria. Health Policy Plan 2016;31:868-77.

20. Lagarde M, Burn S, Lawin L, et al. Exploring the Impact of Performance-Based Financing on Health Workers' Performance in Benin. Washington, DC: World Bank, 2015.

21. Engineer CY, Dale E, Agarwal A, et al. Effectiveness of a pay-forperformance intervention to improve maternal and child health services in Afghanistan: a cluster-randomized trial. Int J Epidemiol 2016;45:451-9.

22. Paul E, Sossouhounto N, Eclou DS. Local stakeholders' perceptions about the introduction of performance-based financing in Benin: a case study in two health districts. Int $J$ Health Policy Manag 2014;3:207-14

23. Chimhutu V, Songstad NG, Tjomsland M, et al. The inescapable question of fairness in Pay-for-performance bonus distribution: a qualitative study of health workers' experiences in Tanzania. Global Health 2016;12:77.

24. Bertone MP, Lagarde M, Witter S. Performance-based financing in the context of the complex remuneration of health workers: findings from a mixed-method study in rural Sierra Leone. BMC Health Serv Res 2016;16:286.

25. Falisse J-B, Meessen B, Ndayishimiye J, et al. Community participation and voice mechanisms under performance-based financing schemes in Burundi. Tropical Medicine \& International Health 2012;17:674-82.

26. Mayumana I, Borghi J, Anselmi L, et al. Effects of Payment for Performance on accountability mechanisms: Evidence from Pwani, Tanzania. Soc Sci Med 2017;179:61-73.

27. Antony M, Bertone MP, Barthes O. Exploring implementation practices in results-based financing: the case of the verification in Benin. BMC Health Serv Res 2017;17:204

28. Binyaruka P, Borghi J. Improving quality of care through payment for performance: examining effects on the availability and stock-out of essential medical commodities in Tanzania. Trop Med Int Health 2017;22:92-102

29. Blaise $\mathrm{P}$, Marchal $\mathrm{B}$, Lefèvre $\mathrm{P}$, et al. Au-delà des méthodes expérimentales: I'approche réaliste en évaluation: Réduire les inégalités sociales en santé, 2010.

30. Chen HT. The roots of theory-driven evaluation. Current views and origins. Evaluation Roots Thousand Oaks, Calif: Sage, 2004.

31. Shepperd S, Lewin S, Straus S, et al. Can we systematically review studies that evaluate complex interventions? PLoS Med 2009;6:e1000086.

32. Petticrew M. Systematic reviews in public health: old chestnuts and new challenges. Bull World Health Organ 2009;87:163-A.

33. White DG. Evaluating evidence and making judgements of study quality: Loss of evidence and risks to policy and practice decisions. Crit Public Health 2001;11:3-17.

34. Pawson R, Tilley N. Realistic evaluation. London, California and New Delhi: Sage, 1997.

35. Julnes G, Mark MM. Evaluation as sensemaking: Knowledge construction in a realist world. New Directions for Evaluation 1998;1998:33-52.

36. Coles $\mathrm{E}$, Wells M, Maxwell M, et al. The influence of contextual factors on healthcare quality improvement initiatives: what works, for whom and in what setting? Protocol for a realist review. Syst Rev 2017;6:168

37. Pawson R, Greenhalgh T, Harvey G, et al. Realist review--a new method of systematic review designed for complex policy interventions. J Health Serv Res Policy. 2005;10 (Suppl 1):21-34

38. Deci EL, Ryan RM. Intrinsic motivation and self-determination in human behavior. New York: Plenum, 1985.

39. Adams JS. Toward an understanding of inequity. J Abnorm Psychol 1963;67:422-36.

40. Vroom VH. Work and motivation. San Francisco, CA.: Jossey-Bass, 1964. 
41. Grossman M. On the Concept of Health Capital and the Demand for Health. J Polit Econ 1972:80:223-55.

42. Anselmi L, Binyaruka P, Borghi J. Understanding causal pathways within health systems policy evaluation through mediation analysis: an application to payment for performance (P4P) in Tanzania. Implement Sci 2017;12:10.

43. Meessen B, Kashala JP, Musango L. Output-based payment to boost staff productivity in public health centres: contracting in Kabutare district, Rwanda. Bull World Health Organ 2007:85:108-15.

44. Gertler PJ, Martinez S, Celhay P. Impact Evaluation of Maternal Child Provincial Health Investment Project in Argentina - Plan Nacer. 2011.

45. Eijkenaar F, Emmert M, Scheppach M, et al. Effects of pay for performance in health care: a systematic review of systematic reviews. Health Policy 2013;110(2-3):115-30.

46. Binyaruka P, Patouillard E, Powell-Jackson T, et al. Effect of Paying for Performance on Utilisation, Quality, and User Costs of Health Services in Tanzania: A Controlled Before and After Study. PLoS One 2015;10:e0135013.

47. Chimhutu V, Lindkvist I, Lange S. When incentives work too well: locally implemented pay for performance (P4P) and adverse sanctions towards home birth in Tanzania - a qualitative study. BMC Health Serv Res 2014;14:23.

48. Bertone MP, Meessen B. Studying the link between institutions and health system performance: a framework and an illustration with the analysis of two performance-based financing schemes in Burundi. Health Policy Plan 2013;28.

49. Renmans D, Holvoet N, Criel B, et al. Performance-based financing: the same is different. Health Policy Plan 2017;32:860-8.

50. Falisse JB, Ndayishimiye J, Kamenyero V, et al. Performance-based financing in the context of selective free health-care: an evaluation of its effects on the use of primary health-care services in Burundi using routine data. Health Policy Plan 2015;30:1251-60.

51. Alonge $\mathrm{O}$, Lin $\mathrm{S}$, Igusa $\mathrm{T}$, et al. Improving health systems performance in low- and middle-income countries: a system dynamics model of the pay-for-performance initiative in Afghanistan. Health Policy Plan 2017;32:1417-26.

52. Ireland M, Paul E, Dujardin B. Can performance-based financing be used to reform health systems in developing countries? Bull World Health Organ 2011;89:695-8.

53. Kalk A, Paul FA, Grabosch E. 'Paying for performance' in Rwanda: does it pay off? Trop Med Int Health 2010;15:182-90.

54. Witter S, Toonen J, Meessen B, et al. Performance-based financing as a health system reform: mapping the key dimensions for monitoring and evaluation. BMC Health Serv Res 2013;13:367.

55. Renmans D, Holvoet N, Orach CG, et al. Opening the 'black box' of performance-based financing in low- and lower middleincome countries: a review of the literature. Health Policy Plan 2016;31:1297-309.

56. Bank W. Country and Lending Groups: The World Bank: data worldbank org, 2015.

57. Pawson R. Evidence-based policy: A realist perspective: Sage publications, 2006

58. Pluye P, Robert E, Cargo M, et al. Proposal: A mixed methods appraisal tool for systematic mixed studies reviews. $2011 \mathrm{http}: / /$ mixe dmethodsappraisaltoolpublic.pbworks.com (Retrieved on 6th Dec 2017)

59. Wong G, Greenhalgh T, Westhorp G, et al. RAMESES publication standards: realist syntheses. BMC Med 2013;11:21.

60. Molnar A, O'Campo P, Ng E, et al. Protocol: realist synthesis of the impact of unemployment insurance policies on poverty and health. Eval Program Plann 2015;48:1-9. 